\title{
Adult onset still's disease masquerading as acute coronary syndrome
}

\author{
Divya Jayakumar, Julia Ash, Stephen A Lobo, Suneesh Anand, Priya Prakash, Anthon R Fuisz, Gregg M Lanier \\ Westchester Medical Center University Hospital, United States
}

Received: August 9, 2016

Accepted: September 11, $2016 \quad$ Online Published: September 17, 2016

DOI: $10.5430 /$ ijdi.v4n1p10

URL: http://dx.doi.org/10.5430/ijdi.v4n1p10

\begin{abstract}
Adult onset still's disease (AOSD) is a rare inflammatory disorder characterized by high fevers, arthralgia, salmon rash and leukocytosis. It is a diagnosis of exclusion and popularly diagnosed by the Yamaguchi criteria. Cardiac involvement in AOSD causing myocarditis is seldom seen. Echocardiography is an important measure of cardiac manifestation, but a more characteristic confirmation is obtained by the cardiac MRI. It is an increasingly recognized noninvasive alternative to the gold standard endomyocardial biopsy. In this case, mid myocardial enhancement in the absence of left ventricular dysfunction was consistent with a diagnosis of myocarditis in AOSD. The delay in diagnosis was caused by AOSD masquerading as acute coronary syndrome. This case highlights the importance of early recognition of myocarditis in an atypical presentation of AOSD which results in early immunomodulatory therapy and an improved clinical outcome.
\end{abstract}

Key Words: Myocarditis, Still's disease, Cardiac magnetic resonance imaging, Mid-myocardial enhancement

\section{INTRODUCTION}

Adult onset still's disease (AOSD) is a rare inflammatory disorder characterized by a spectrum of high fevers, arthralgia, evanescent rash and leukocytosis. ${ }^{[1]}$ It usually mimics an infectious process because of fever and leukocytosis. In the case presented, the delay in diagnosis was attributed to AOSD masquerading as an acute coronary syndrome. AOSD is a diagnosis of exclusion and cardiac involvement causing myocarditis is seldom seen. Echocardiography is an important screening tool to evaluate cardiac manifestations of AOSD, but more characteristic confirmation of myocardial involvement can be obtained by cardiac magnetic resonance imaging (MRI). Cardiac MRI is an increasingly recognized non-invasive alternative to endomyocardial biopsy in the evaluation of myocardial disease. Cardiac MRI has a high specificity $(91 \%)$ in the diagnosis of myocarditis, without requiring an invasive endomyocardial biopsy. ${ }^{[2]}$ We describe a case of AOSD presenting as acute coronary syndrome with subsequent diagnosis of myocarditis established by Cardiac MRI. This case highlights the importance of early recognition of myocarditis in an atypical presentation of AOSD which resulted in early immunomodulatory therapy and an improved clinical outcome.

\section{Case presentation}

This is a 49-year-old Caucasian woman with a past medical history of recurrent sinusitis and fibromyalgia who presented to an outside community hospital with a 2-month history of intermittent fevers associated with fatigue, myalgia, sore throat, diffuse joint pain and an unintentional 20pound weight loss. Prior to admission, she was treated with doxycycline for presumed Lyme disease, without relief of symptoms.

*Correspondence: Divya Jayakumar; Email: divyajk87@gmail.com; Address: Westchester Medical Center University Hospital, United States. 
Physical examination showed high grade fever, pallor, evanescent macular non-blanching rash on abdomen and tenderness of bilateral forearms and thighs, without synovitis. Initial laboratory tests were significant for WBC of 19,000 cells/mcl, microcytic anemia with a hemoglobin of $7.9 \mathrm{mg} / \mathrm{dl}$, platelets of $121,000 / \mathrm{mcl}$ and mild transaminitis. Chest radiograph was significant for bilateral opacities. Computed tomography of chest and abdomen showed ground glass opacities, bilateral pleural effusions (see Figure 1), mediastinal lymphadenopathy, supraclavicular necrotic lymph nodes and hepatosplenomegaly (see Figures 2, 3). The infectious workup for viral, bacterial, parasitic, mycobacterial and tick borne diseases was negative. Bone marrow biopsy revealed a hypercellular myeloid marrow with increased iron stores, but no evidence of lymphoma or leukemia.

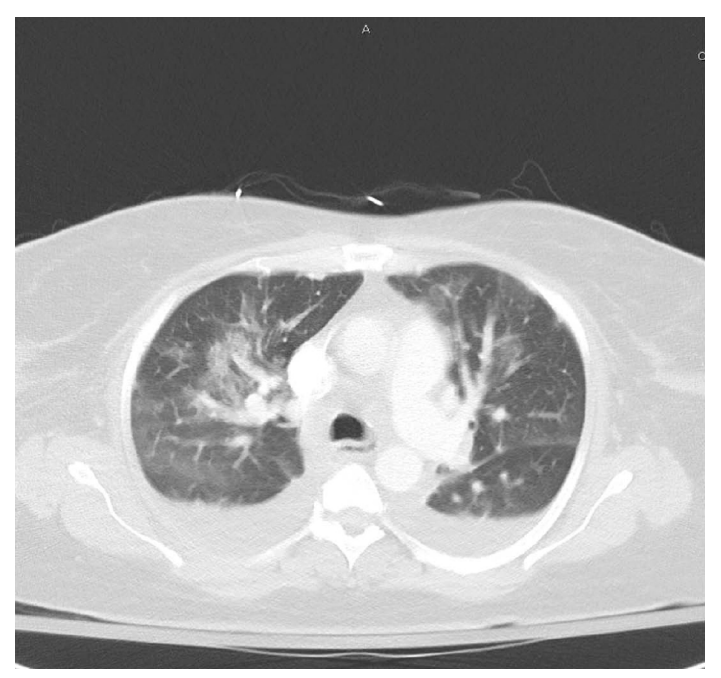

Figure 1. CT chest showing ground glass opacities, bilateral pleural effusions, mediastinal lymphadenopathy

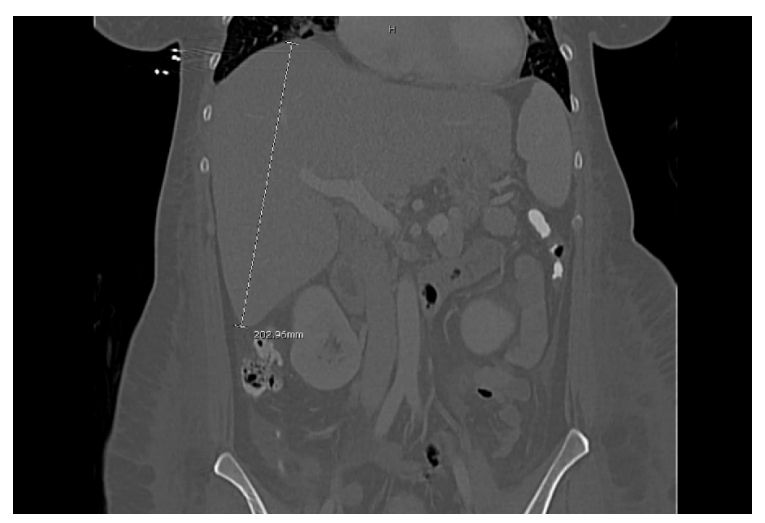

Figure 2. CT abdomen showing hepatomegaly of $20.2 \mathrm{~cm}$

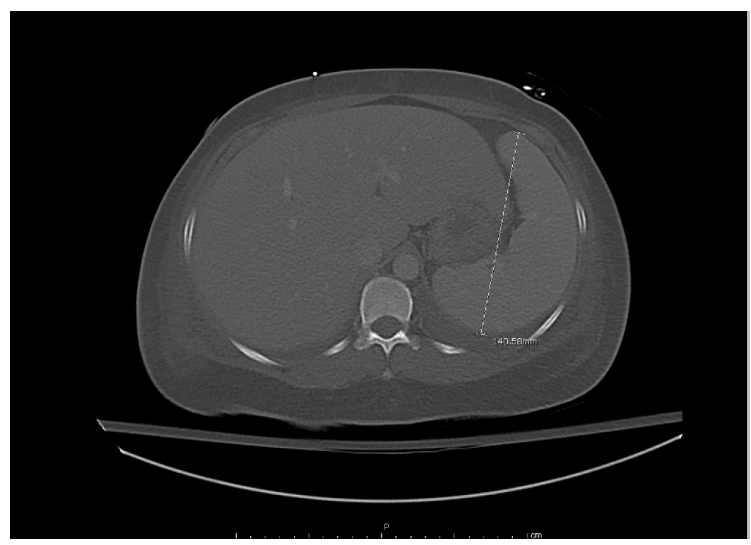

Figure 3. CT abdomen showing splenomegaly of $14 \mathrm{~cm}$

The patient continued to spike fevers and developed hypotension for which she was started on broad spectrum antibiotics and vasopressors for presumed septic shock. A supraclavicular lymph node biopsy was performed which showed benign reactive pathology. Soon after the lymph node biopsy, she developed mid-sternal chest pain and an electrocardiogram (ECG) showing new ST depression in inferior and anterolateral leads, as compared with baseline ECG (see Figure 4). The troponin I was $21.93 \mathrm{ng} / \mathrm{ml}$ at the time of chest pain, and she was treated for possible non-ST elevation myocardial infarction (NSTEMI) with heparin drip, aspirin and clopidogrel. Echocardiography showed inferior basilar hypokinesis with diastolic dysfunction, grossly normal ejection fraction and no valvular abnormalities. Because of chest pain, positive troponin and regional wall motion abnormalities, she was transferred to our facility for diagnostic and possibly therapeutic cardiac catheterization.

Upon transfer to our hospital, alternative diagnoses were considered before proceeding with a cardiac catheterization and biopsy. Laboratory tests revealed a highly elevated erythrocyte sedimentation rate of $86 \mathrm{~mm} / \mathrm{hr}, \mathrm{C}$ reactive protein of $20 \mathrm{mg} / \mathrm{L}$, ferritin of $248 \mu \mathrm{g} / \mathrm{L}$ and normal complement levels. Rheumatologic workup including anti-nuclear antibody (ANA), anti neutrophil cytoplasmic antibody (ANCA), anti cyclic citrullinated peptide antibody (CCP), anti smooth muscle antibody, anti double stranded DNA antibody and rheumatoid factor (RF) were normal. Given the patient's atypical cardiac symptoms with regional wall motion abnormality on echocardiogram, a cardiac MRI was obtained for further evaluation. The gadolinium enhanced cardiac MRI showed mid-myocardial enhancement, a finding consistent with myocarditis in AOSD $^{[3]}$ (see Figure 5), which shows cardiac MRI showing mid-myocardial enhancement. 


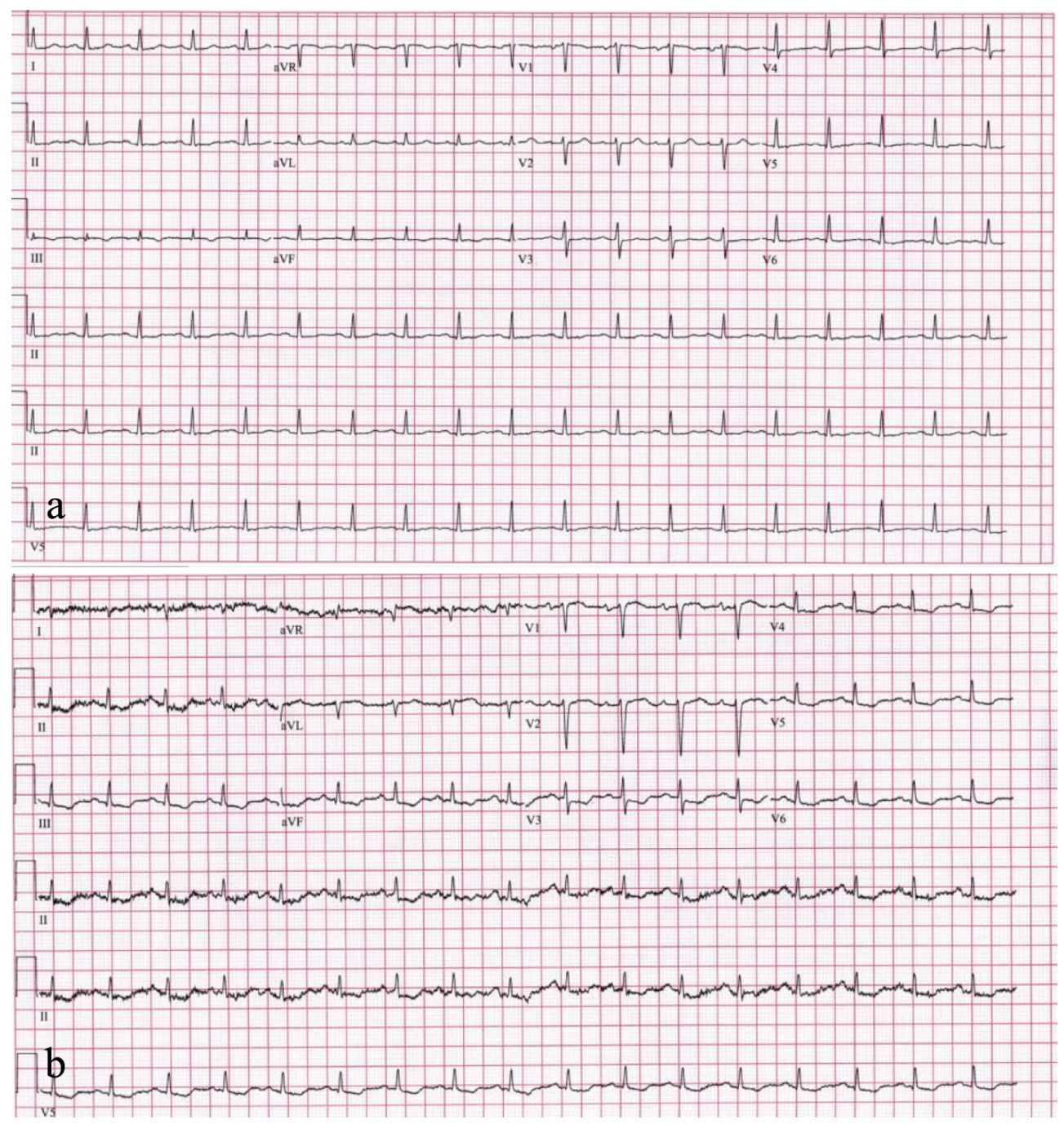

Figure 4. a. Initial ECG prior to lymph node biopsy showing sinus tachycardia; b. Subsequent ECG after lymph node biopsy showing sinus tachycardia, ST depressions in inferior and anterior leads, possible anterolateral and inferior ischemia

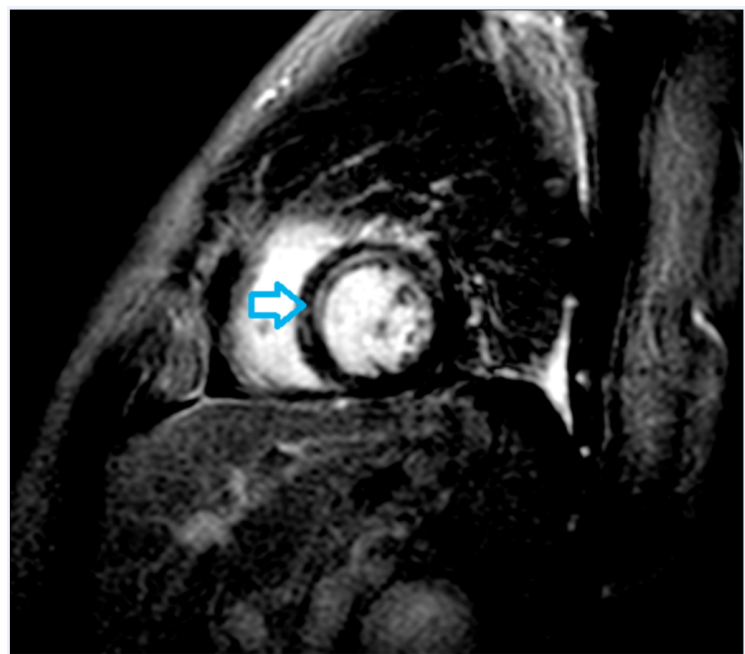

Figure 5. Cardiac MRI showing mid-myocardial enhancement
A diagnosis of AOSD was made as per Yamaguchi criteria and she was started on prednisone $0.5 \mathrm{mg} / \mathrm{kg} /$ day with significant clinical and laboratory improvement. As a result of clinical diagnosis and cardiac MRI findings, cardiac catheterization was not pursued. She was asymptomatic on discharge without fever, myalgia, rash or ECG changes.

\section{DisCUSSION}

The term "Adult Onset Still's disease" was first used by Bywaters in 1971 to describe adults with clinical characteristics similar to systemic juvenile idiopathic arthritis who did not fulfill the criteria for rheumatoid arthritis. ${ }^{[4]}$ Our 49-year-old lady fulfilled all 9 of the popularly used Yamaguchi criteria for AOSD, namely leukocytosis, fever, arthralgia, rash, sore throat, lymphadenopathy, hepatosplenomegaly, transaminitis and negative ANA and RF. This rare disease has a prevalence of less than 1 case per 100,000 persons. ${ }^{[2]}$ It affects 
males and females equally, and majority fall in the age group below 35 years. ${ }^{[5]}$ It is a diagnosis of exclusion and is diagnosed after ruling out infectious, neoplastic and autoimmune etiologies. [6]

The clinical course of AOSD falls into three main categories - Monophasic, intermittent and chronic. ${ }^{[7,8]}$ Despite lack of data, AOSD associated with myocarditis tends to follow a monophasic or chronic pattern. ${ }^{[2]}$ Pathophysiology of myocarditis in Still's disease is uncertain. Proposed theories involve inflammatory response resulting in lymphocytic infiltration which may lead to fibrosis and atrophy. ${ }^{[2]}$ Cardiac involvement, especially myocarditis is rare and can be identified by cardiac enzymes, ECG, cardiac MRI and endomyocardial biopsy.

Cardiac manifestations in AOSD are uncommon and include pericarditis, pericardial effusion, myocarditis, cardiomyopathy and conduction abnormalities. Pericarditis has been reported in up to $20 \%$, while myocarditis is fairly infrequent and has been noted only in 3\%-7\%..$^{[2,9,10]}$ Myocarditis has been found to have a male predominance, with symptoms of shortness of breath, chest pain or palpitations. ${ }^{[11]}$ Despite having serious consequences, cardiac involvement has not been associated with poor prognosis when treated in a timely manner. ${ }^{[12]}$ In our case, the delay in diagnosis was attributed to myocarditis manifesting as the presenting symptom of Still's disease. Cardiac involvement does not usually manifest as the presenting symptom, though myocarditis seems to be an early complication occurring in the first year of the disease. ${ }^{[2]}$ It can complicate the disease further by triggering

\section{REFERENCES}

[1] Yamaguchi M, Ohta A, Tsunematsu T, et al. Preliminary criteria for classification of adult still's disease. J Rheumatol. 1992; 19(3): 424-30. PMid: 1578458

[2] Gerfaud-Valentin M, Seve P, Iwaz J, et al. Myocarditis in adultonset still disease. Medicine (Baltimore). 2014; 93(17): 2809. PMid:25398063 http://dx.doi.org/10.1097/MD.000000 0000000112

[3] Yamazoe M, Mizuno A, Suyama Y, et al. Endomyocardial biopsy and magnetic resonance imaging of acute myocarditis with adult-onset still's disease. Korean Circ J. 2014; 44(6): 437-40. PMid:25469148 http://dx.doi.org/10.4070/kcj .2014.44.6.437

[4] Bywaters EG. Still's disease in the adult. Ann Rheum Dis. 1971; 30(2): 121-33. http://dx.doi.org/10.1136/ard.30.2.121

[5] Efthimiou P, Paik PK, Bielory L. Diagnosis and management of adult onset still's disease. Ann Rheum Dis. 2006; 65(5): 56472. PMid:16219707 http://dx.doi.org/10.1136/ard. 2005. 042143

Published by Sciedu Press complete heart block, tachyarrhythmia, cardiac tamponade, heart failure and cardiogenic shock.

The array of non-specific symptoms and lack of definite serological markers makes Still's disease a challenge for physicians. This patient's chest pain, positive cardiac enzymes and echocardiography showing regional hypokinesis initially suggested a more common diagnosis, NSTEMI from coronary artery disease, rather than an autoimmune etiology. In this case, cardiac MRI abrogated the need for an invasive cardiac catheterization or endomyocaridal biopsy. Based on the work by Friedrich M et al., myocarditis was diagnosed by the Lake Louise Criteria. ${ }^{[13]}$ Cardiac MRI with gadolinium showed mid- myocardial enhancement, consistent with similar findings in limited case reports of Still's disease ${ }^{[3]}$ (see Figure 5).

The first line of treatment of Still's disease are non-steroidal anti-inflammatory drugs (NSAIDS). Patients who are refractory or intolerant to NSAIDs or have severe disease, are treated with corticosteroids, immunosuppressants or biologics. In our case, the patient's systemic symptoms and myocarditis responded well to prednisone $0.5 \mathrm{mg} / \mathrm{kg} / \mathrm{day}$. Myocarditis is a potentially life threatening condition which necessitates prompt recognition and close monitoring for ventricular arrhythmias and heart block. The timely recognition of myocarditis as a presenting symptom of AOSD and subsequent treatment in this case was facilitated by the characteristic findings seen on cardiovascular MRI.

\section{CONFlicts of InTEREST Disclosure}

The authors have declared no conflicts of interest.
[6] Gopalarathinam R, Orlowsky E, Kesavalu R, et al. Adult onset still's disease: A review on diagnostic workup and treatment options. Case Rep Rheumatol. 2016; 2016: 6502373. http://dx.doi.org/10. $1155 / 2016 / 6502373$

[7] Gerfaud-Valentin M, Maucort-Boulch D, Hot A, et al. Adultonset still disease: Manifestations, treatment, outcome, and prognostic factors in 57 patients. Medicine (Baltimore). 2014; 93(2): 91-9. PMid:24646465 http://dx.doi.org/10.1097/MD.0000 000000000021

[8] Kontzias A, Efthimiou P. Adult-onset still's disease: Pathogenesis, clinical manifestations and therapeutic advances. Drugs. 2008; 68(3): 319-37. http://dx.doi.org/10.2165/00003495-200 868030-00005

[9] Drouot MH, Hachulla E, Houvenagel E, et al. Cardiac complications in adult onset still disease: From pericarditis to tamponade as manifestations. Rev Med Interne. 1994; 15(11): 740-3. http://dx.doi.org/10.1016/S0248-8663(05) 81400-6

[10] Moder KG, Miller TD, Allen GL. Cardiac tamponade: An unusual feature of adult onset still's disease. J Rheumatol. 1995; 22(1): 180-2. 
PMid:7699669

[11] Caforio AL, Calabrese F, Angelini A, et al. A prospective study of biopsy-proven myocarditis: Prognostic relevance of clinical and aetiopathogenetic features at diagnosis. Eur Heart J. 2007; 28(11) 1326-33. PMid:17493945 http://dx.doi.org/10.1093/eurhe $\operatorname{artj/ehm076}$

[12] Parvez N, Carpenter JL. Cardiac tamponade in still disease: A review of the literature. South Med J. 2009; 102(8): 832-7. PMid:19593288 http://dx.doi.org/10.1097/SMJ.0b013e3181ad4847

[13] Friedrich MG, Sechtem U, Schulz-Menger J, et al. International Consensus Group on Cardiovascular MR in Myocarditis. Cardiovascular magnetic resonance in myocarditis: A JACC white paper. J Am Coll Cardiol. 2009; 53(17): 1475-87. PMid:19389557 http://dx.doi.org/10.1016/j.jacc.2009.02.007 